\title{
Germanica
}

\section{Arthur Schnitzler und Tristan Bernard : Anmerkungen zu einem singulären Fall von 〈Doppelgängerschaft〉}

Arthur Schnitzler et Tristan Bernard : remarques sur un cas singulier de "double littéraire»

Arthur Schnitzler and Tristan Bernard : observations on a singular case of a "literary double"

Wolfgang Lukas

\section{OpenEdition}

Journals

Édition électronique

URL : http://journals.openedition.org/germanica/2123

DOI : $10.4000 /$ germanica. 2123

ISSN : 2107-0784

Éditeur

Université de Lille

Édition imprimée

Date de publication : 15 juin 2013

Pagination : 85-99

ISBN : 9782913857315

ISSN : 0984-2632

\section{Référence électronique}

Wolfgang Lukas, « Arthur Schnitzler und Tristan Bernard: Anmerkungen zu einem singulären Fall von 〈Doppelgängerschaft〉 », Germanica [Online], 52 | 2013, Online erschienen am: 26 Juni 2013, abgerufen am 06 Oktober 2020. URL : http://journals.openedition.org/germanica/2123 ; DOI : https://doi.org/ 10.4000/germanica.2123 


\title{
Arthur Schnitzler und Tristan Bernard : Anmerkungen zu einem singulären Fall von〈Doppelgängerschaft〉
}

\author{
Wolfgang LUKAS \\ Bergische Universität Wuppertal
}

\begin{abstract}
Die frappierende Ähnlichkeit zwischen Tristan Bernards Boulevardstück Les deux canards (1913) und Schnitzlers Komödie Fink und Fliederbusch (1917) stellt einen erstaunlichen - und in Schnitzlers Werk singulären - Fall von augenscheinlicher 〈Doppelgängerschaft〉 dar, die nicht auf Rezeption oder 〈Einfluss〉 zurückführbar ist. Der Beitrag unternimmt den Versuch, gemeinsame Konstellationen jenseits der offenkundigen Journalistensatire zu rekonstruieren, auf deren Hintergrund beide Stücke als je individuelle Ausprägungen einer zeitgenössischen Grundproblematik erscheinen.
\end{abstract}

Am 12. Dezember 1913 erfährt Arthur Schnitzler bei einer abendlichen Einladung bei Berta Zuckerkandl von einem neuen französischen Theaterstïck des seinerzeit sehr populären Bühnenautors Tristan Bernard (1866-1947), Les deux canards $^{1}$, das in frappanter Weise dem sog. « Journalistenstück » ähnelt, an dem er selbst in dieser Zeit arbeitet :

1. - Die Uraufführung des Stücks, für welches auch Alfred Athis als Ko-Autor zeichnet, fand am 3.12.1913 im Théâtre du Palais-Royal statt; die deutsche Erstaufführung in Berlin am 1.3.1914 u.d.T. Er und der Andere (Übs. v. Max Schönau).

GERMANICA, 2013, LII, pp. 85 à 99. 
Nach dem Souper erzählt die Hofrätin von dem Erfolg eines neuen Stücks Bernard's in Paris, erzählt den Inhalt... „Ein Journalist X... an einem radic. Blatt -, ein Y an einem Aristokratenblatt - es stellt sich heraus - dass sie - eine Person sind - die Feindschaft - entwickelt sich weiter - bis er sich mit sich selber duelliren muss -“ Ich - erkläre: mein Stück - dessen Scenarium so gut wie fertig! - in der Grundlinie absolut identisch. Allgemeine Verblüffung. Aber ich solle das Stück doch schreiben ${ }^{2}$.

Schnitzler reagiert auf diesen Schock mit einer förmlichen Erklärung, in der er die Alleinautorschaft an seinem Text versichert und auf das merkwürdige Phänomen einer Übereinstimmung zweier gleichzeitig, aber unabhängig voneinander entstehender literarischer Texte abhebt :

Mit diesen Zeilen (die ich eventuell der Buchausgabe meiner Komödie vorausschicke) will ich nicht so sehr eine Priorität oder Gleichzeitigkeit feststellen, an der auch auf mein Wort hin niemanden [!] zu zweifeln einfallen dürfte, sondern vor allem ein ziemlich merkwürdiges Beispiel für die Tatsache aufbewahren, dass in zwei Schriftstellern, die einander nie gesehen, nie gesprochen haben und jeder in einem anderen Lande leben, ein im Grunde ziemlich neuer Bühneneinfall fast zur selben Zeit reif wird und nach Gestaltung drängt. Dass es mir beschieden war dieses Abenteuer gerade mit Tristan Bernard zusammen zu erleben, gereicht mir schon darum zur Genugtuung, weil ich gerade diesem ausserordentlichen Humoristen seit Jahren eine ganz besondere Verehrung entgegenbringe ${ }^{3}$.

Die bisher verfassten Manuskripte und Typoskripte lässt er von seiner Sekretärin dreimal abschreiben und bei drei verschiedenen Personen seines Vertrauens deponieren; der Bucherstausgabe von Fink und Fliederbusch bei S. Fischer 1917 wird dann allerdings keinerlei Bemerkung vorangestellt.

Tatsächlich ist die Ähnlichkeit der beiden Komödien frappant und führt reflexhaft zur Vermutung, dass von einer der beiden Seiten aus irgendeine Rezeption, und sei sie noch so halbbewusster Art, stattgefunden haben müsse - eine Vermutung, die hier aber ohne jeden Zweifel negativ zu bescheiden ist. Der Nachlass bewahrt (für die SchnitzlerÜberlieferung ungewöhnlich) reichhaltiges Quellenmaterial auf, das Schnitzler als Anregung gedient hat, wie u.a. Berichte von Sitzungen aus dem Abgeordnetenhaus aus den Jahren 1894 und 1896 sowie Zeitungsartikel über zeitgenössische Pressefehden zwischen reaktionären und sozialdemokratischen Blättern. In Bezug auf Bernards Stück

2. - A. Schnitzler: Tagebuch 1913-1916, hg. v. Werner Welzig, 2. unver. Aufl. Wien, Akademie der Wissenschaften, 1999, S. 82.

3. - Nachlass Cambridge, University Library, Schnitzler Papers, Mappe A 114,1. 
gibt es für uns somit keinen Anlass, an Schnitzlers Versicherung der absoluten Eigenständigkeit seiner Komposition zu zweifeln, wie sehr die Beziehungen seines Bühnenwerks zum französischen Boulevardtheater auch ansonsten außer Frage stehen ${ }^{4}$.

Eine Literaturwissenschaft, die primär in der Kategorie von 〈Einfluss〉 denkt, gerät hier zwangsläufig in eine Notlage, da sie dieses 〈Doppelgänger〉-Phänomen nicht erklären kann. Angesichts des nur zu offensichtlichen Komplexitätsunterschieds beider Stücke bietet sich als Ausweg eine Inkommensurabilitätsbehauptung an, die a priori einer vergleichenden Analyse enthebt ${ }^{5}$. Im Folgenden sei hier stattdessen der Versuch einer Analyse unternommen, die sich jenseits der Ebene der Beeinflussung ${ }^{6}$ und damit auch der intentionalen Zurechenbarkeit situiert. Zu rekonstruieren ist eine abstrakte, über die Journalismusthematik hinausgehende gemeinsame Problemkonstellation, die im Kontext zeitgenössischer denk- und mentalitätsgeschichtlicher Strömungen zuallererst ihre Bedeutung erhält. An die Stelle einer einseitigen kausalen Relation würden dergestalt wechselseitige homologe Relationen treten, die beide Stücke als je individuelle Ausprägung einer identischen zugrundeliegenden Thematik erscheinen ließen.

4. - Siehe hierzu und generell zum Kontext der zeitgenössischen Dramatik die Studie von Wolfgang Sabler: Arthur Schnitzler. Écriture et conventions théâtrales, Bern u.a., Lang, Collection Contact, Série I - Theatrica, 21, 2002.

5. - So Françoise Derré: «Une curieuse interférence : Les Journalistes et Les Deux Canards », in : Jacques Le Rider/Renée Wentzig (Hg.) : «Les Journalistes » de Arthur Schnitzler. Satire de la presse et des journalistes dans le théâtre allemand et autrichien contemporain, Tusson 1995, S. 250-262.

6. - Die es auch hier natürlich gibt, wobei die Suche nach möglichen Prätexten bzw. Inspirationsquellen bereits zu Schnitzlers Zeiten einsetzte. Neben Freytags Die Journalisten (hierzu v.a. Jacques Le Rider: «Les Journalistes de Gustav Freytag, protoype de la satire de la presse et des journalistes dans le théâtre de langue allemande », in: Le Rider/Wentzig, a.a.O, S. 263-274) wurde bereits von der zeitgenössischen Kritik auf Emile Augiers Le Fils de Giboyer hingewiesen (s. Sigurd P. Scheichl: « Echoes of Emile Augier in Arthur Schnitzlers Fink und Fliederbusch », in : Austria and France, 2005, S. 77-91, hier 81). Auch das Libretto zu Jacques Offenbachs Orpheus in der Unterwelt von Henri Crémieux (in der mutmaßlichen Übs. von Johann Nestroy) hat man namhaft gemacht sowie sogar zwei Stücke aus Romantik und Biedermeierzeit : s. G. J. Weinberger : Arthur Schnitzler's Late Plays. A Critical Study. New York 1997, Kap. 3 «Fink und Fliederbusch: The Identity of Opposites », S. 49-84, hier 49f.; Ernst L. Offermanns : «Arthur Schnitzlers Komödie Fink und Fliederbusch », in : Modern Austrian Literature 3, 1970, S. 5-24, hier 8 ; Hartmut Scheible : « 〈Je sais écrire pour n'importe quelle tendance>. Les comédies de journalistes avant Schnitzler », in: Le Rider/Wentzig, a.a.O, S. 13-71 ; Reinhard Urbach : Arthur Schnitzler, 2. Aufl. Velber 1972, S. 91. 


\section{Ideologisierung und 〈Weltanschauung〉}

Beide Stücke sind von der Gattung her zunächst satirische Journalistenkomödien, die jeweils bestimmte zeitgenössische Zustände, nämlich die Tendenzen einer zunehmenden Politisierung und weltanschaulichen Ideologisierung im Umkreis des Ersten Weltkriegs aufs Korn nehmen, wobei beide Stücke auch einen expliziten lokalen bzw. regionalen Bezug aufweisen : die französische Provinz im Falle Bernards, Wien im Falle Schnitzlers 7 . Die Gemeinsamkeiten finden sich sowohl hinsichtlich der statisch-semantischen (wie u.a. Figurenkonstellation und Raumsemantik) als auch der dynamisch-narrativen Textdimension.

Ort des Geschehens bei Bernard ist das fiktive Provinzstädtchen Valmoutiers, wo sich zwei Provinzblätter, die titelgebenden « canards », befehden: Das eine Blatt, Le Phare, wird von Figuren bürgerlichen Standes gemacht und artikuliert <linke〉, gesellschaftskritische bis republikanisch-revolutionäre Positionen; das andere, La Torche, wird von Aristokraten herausgegeben und vertritt systemaffirmative bis rechtsreaktionäre Positionen und ist im Prinzip völlig unpolitisch : Hier werden primär Artikel über aristokratische Mußebeschäftigungen wie Jagd, Fliegerei, Tanz und Mode verfasst, noch dazu von sprachlich höchst fragwürdiger Gestalt. Bei Schnitzler befehden sich die liberal-demokratische Gegenwart, ein bürgerliches Blatt, und die konservativ-reaktionäre Elegante Welt, die in höchsten gesellschaftlichen Kreisen - Hof, Adel, Klerus und « haute Finance »- gelesen wird ; auch hier zunächst ein « Klatschblatt », das schwerpunktmäßig über « Geschichten aus der Kulissenwelt » berichtet, dessen « Politisierung » aber nun betrieben wird (FF, 571, 596, 606) ${ }^{8}$.

Die politisch-ideologische Opposition wird jeweils in einer räumlichtopographischen Opposition gespiegelt, und beide Stücke entwerfen eine diesbezüglich homologe Raumsemantik. Während die bürgerlichdemokratische Zeitung in einem üblichen Redaktionsbüro bzw. einem Raum mit einer für diese Zwecke erwartbaren Einrichtung situiert wird (bei Bernard in der Druckerei), finden die Redaktionssitzungen des oppositionellen Blatts faktisch oder tendenziell in einem Privatraum statt : im Falle Bernards in der mit Bergère und Piano ausgestatteten Halle im außerhalb der Stadt gelegenen Schloss des Barons St.-Amour; im Falle Schnitzlers zwar ein « Redaktionslokal », das aber ausweis-

7. - Zur satirischen Dimension s. den von J. Le Rider und R. Wentzig herausgegebenen thematischen Sammelband (a.a.O.) ; s. auch Sabler, a.a.O., S. 266 und Karl Zieger : «La satire sociale et politique d'Arthur Schnitzler et sa réception (tardive) en France », in : Jeanne Benay et Gilbert Ravy (Hg.), Écritures et langages satiriques en Autriche (1914-1938), Bern, Berlin, etc., Peter Lang, 1999, S. 295-315.

8. - Der Text wird im Folgenden mit der Sigle FF zitiert aus: Arthur Schnitzler: Die dramatischen Werke, Bd. 2, Frankfurt a. M., Fischer, 1962, S. 555-649. 


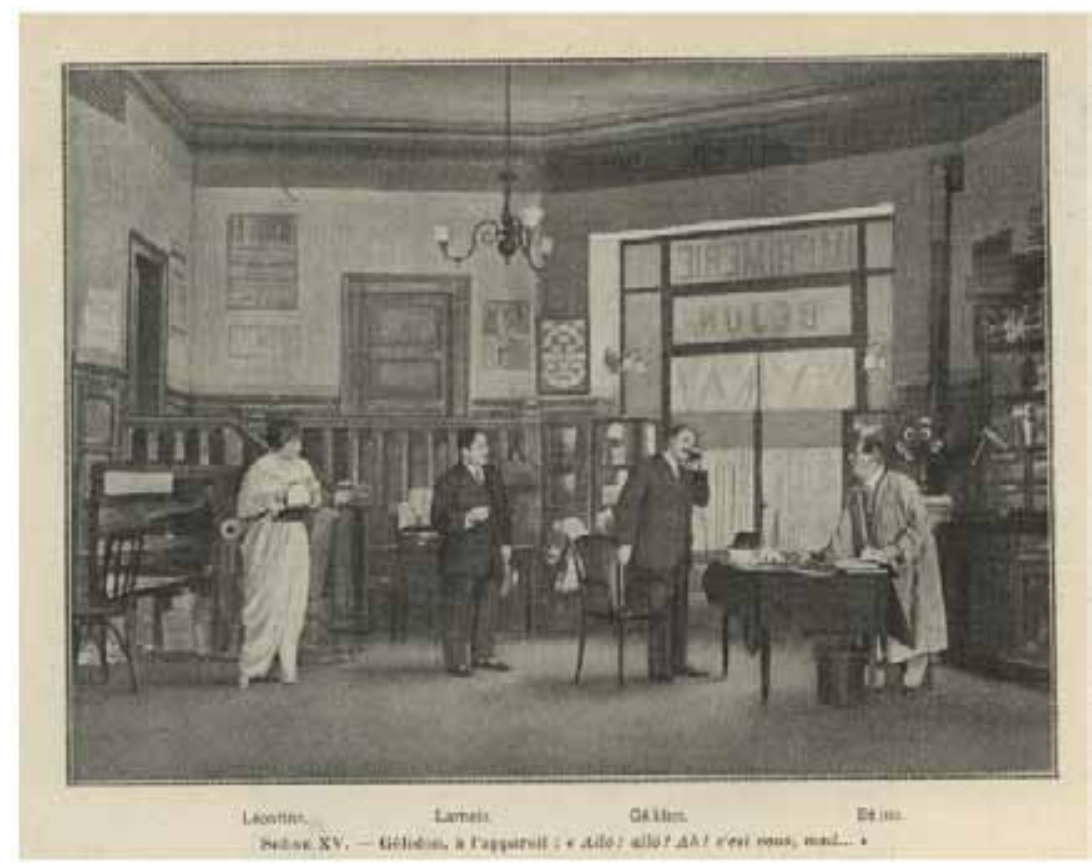

Abb. 1 : Szenenfoto der Pariser Uraufführung von Les deux canards, II. Akt. La Petite Illustration, a.a.O., S. 1.

In der Redaktion der « Gegenwart».

Geräumiges Zimmer. [...] Zwei Schreibtische in der Mitte einander gegenüber mit Tischtelephon. An der rechten und linken Wand vorn je ein Stehpult. Rückwärts zu beiden Seiten der Türe je ein großer Schrank, der eine offen mit Aktenmappen und Zeitungen. Auf den Schränken Faszikel. In den Pultfächern Bücher [...]. Einfacher Messinglüster mit drei Flammen. (FF, 557).

lich des Dekors « eher einem leidlich eleganten Herrenzimmer ähnlich [sieht] »(FF, 586). Abb. 1 und 2 konfrontieren das im Journaldruck in La Petite Illustration wiedergegebene Szenenphoto der Pariser Inszenierung von Les deux canards mit den jeweiligen Nebentexten aus Fink und Fliederbusch.

Held der beiden Stücke ist ein junger unverheirateter Mann, der bei beiden Journalen jeweils unter verschiedenem Namen die entscheidenden Leitartikel verfasst, die das politische Profil prägen und zu einer ideologischen Verschärfung der Gegensätze führen, die im III. Akt schließlich in die komische Zuspitzung eines Duells zwischen dem Helden und seinem 〈Doppelgänger〉 münden. Bei Bernard ist es ein junger Pariser Schriftsteller, der bei beiden Zeitungen als Chefredakteur 
fungiert, beim republikanischen Phare unter seinem echten bürgerlichen Namen Lucien Gélidon, bei der reaktionären Torche unter seinem adligen Pseudonym Henri de Montillac. Schnitzlers Titelheld entstammt gemäß seinen eigenen Angaben kleinbürgerlichem und, wie seine beiden Namen signalisieren, jüdischem Milieu ${ }^{9}$. Homolog ist schließlich auch die raumsequentielle Struktur der beiden Dreiakter, wie nachstehend schematisch zusammengefasst : Auf den im I. Akt gezeigten bürgerlich-demokratischen Innenraum folgt im II. Akt als Kontrast der aristokratische Innenraum, während die Zuspitzung - und gleichzeitige Lösung - der Gegensätze jeweils in einem dritten, einem Außenraum, jeweils einem Schlosspark stattfindet.

\begin{tabular}{|c|c|c|c|}
\hline Akte & I & II & III \\
\hline \multirow{2}{*}{ Les deux canards } & $\begin{array}{c}\text { Redaktionsbüro } \\
\text { «Le Phare } \\
\text { [urspr. «La Torche »] }\end{array}$ & $\begin{array}{c}\text { Redaktionsbüro» } \\
\text { «La Torche }\end{array}$ & $\begin{array}{c}\text { Schlosspark } \\
\text { (Baron St.-Amour) }\end{array}$ \\
\cline { 2 - 4 } & Lucien Gélidon $\leftrightarrow$ & Henri de Montillac & \\
\hline Fink und Fliederbusch & $\begin{array}{c}\text { Redaktionsbüro } \\
\text { «Die Gegenwart » }\end{array}$ & $\begin{array}{c}\text { Redaktionsbüro } \\
\text { «Die elegante Welt » }\end{array}$ & $\begin{array}{c}\text { Schlosspark } \\
\text { (Fürstin Wendolin) }\end{array}$ \\
\cline { 2 - 5 } & Fliederbusch $\leftrightarrow$ & Fink & \\
\hline
\end{tabular}

Die Analogien erstrecken sich darüber hinaus auf weitere Figuren und Handlungssequenzen wie u.a. den Stellen- und damit ideologischen Lagerwechsel von Redakteuren (La Flache bzw. Styx) oder den faktischen (bzw. bei Schnitzler nur geplanten) Kauf der Zeitung - La Torche bzw. Die Elegante Welt - durch einen Adligen, der als Galionsfigur des konservativen Lagers öffentlich auftritt, Baron St-Amour und Graf Niederhof. Diese beiden besetzen nicht nur die analoge strukturelle Position, sondern erhalten sogar annähernd identische Charaktermerkmale. Wird der Baron von Gélidons Freund Larnois (und zugleich auktorial) qualifiziert als « très hautain, très gourmé, mais, au fond, pas si intransigeant qu'il en a l'air » (I, 4 ; DC, 5) $)^{10}$, so charakterisiert Schnitzler im Nebentext den Grafen als « [...] elegant,

9. - Siehe Sigurd P. Scheichl: «Juden, die keine Juden sind. Die Figuren in Schnitzlers Fink und Fliederbusch », in: Mark Gelber u.a. (Hg.): Von Franzos zu Canetti. Jüdische Autoren aus Österreich. Neue Studien, Tübingen 1996, S. 225-238 ; G. J. Weinberger, a.a.O., hier 60f.

10. - Ich zitiere nach dem Erstdruck: Les deux canards. Pièce en trois actes par Tristan Bernard et Alfred Athis. In : La Petite Illustration. Roman - Théâtre. Revue hebdomadaire, $n^{\circ} 57,4$. Avril 1914, p. 1-39, hier S. 9. (Im Folgenden im laufenden Text mit Sigle DC zitiert.) 


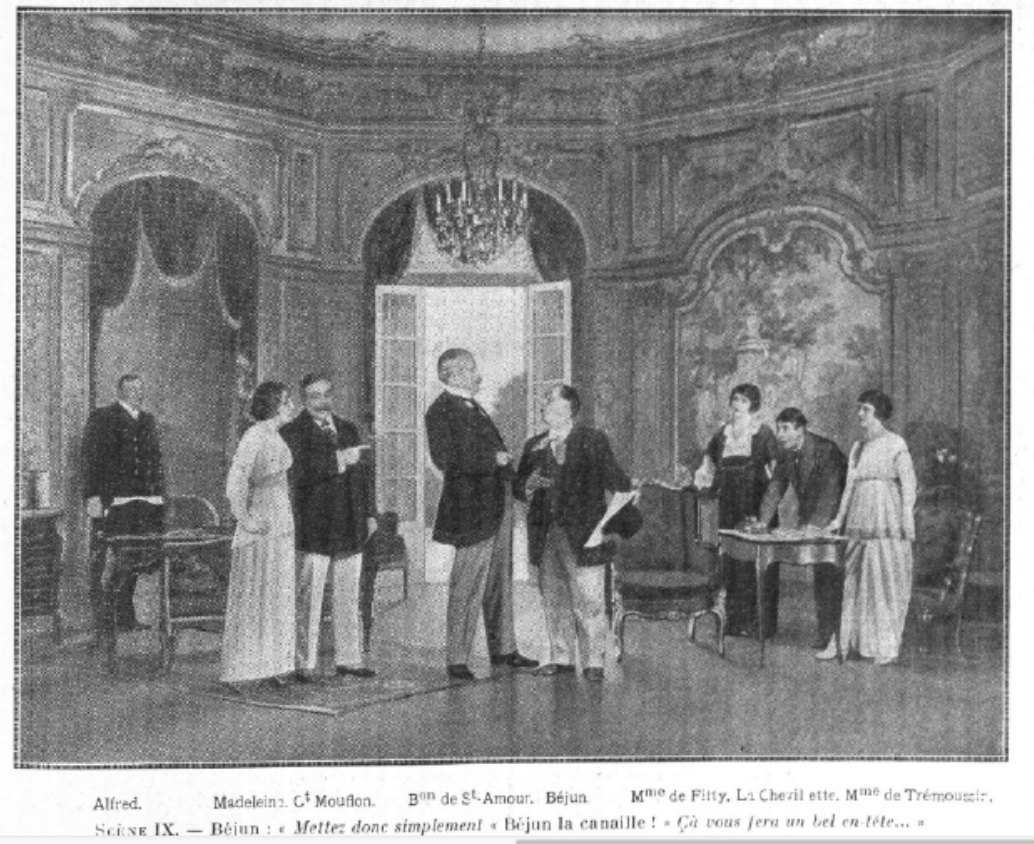

Abb. 2: Szenenfoto, II. Akt. La Petite Illustration, a.a.O., S. 15.

(Photographies : A. Bert. Copyright : Tristan Bernard et Alfred Athis).

Das Redaktionslokal der «Eleganten Welt». Sieht eher einem leidlich eleganten Herrenzimmer ähnlich. Dunkelrote Tapeten. In der Mitte des Zimmers ein nicht sehr großer Schreibtisch. [...] An den Schreibtisch gerückt ein Diwan mit persischem Teppich und vielen Polstern. [...] Links an der Wand Kamin. Darüber ein Spiegel. Rückwärts ein Bücherschrank. Rechts eine Art Aktenschrank, der nicht hereinpaßt und durch einen Vorhang so ziemlich kaschiert ist [...]. (FF, 586).

von einer zuweilen recht hochmütigen Liebenswürdigkeit » (FF, 593). Gemeint ist der - in beiden Stücken jeweils durchaus positiv zu verstehende - Hochmut desjenigen, der über den Lagern bzw. Parteien steht, auch wenn er vordergründig Repräsentant des einen - konservativen Lagers ist.

Denn von beiden Stücken wird die ideologische Oppositionsbildung gründlich destruiert. Von Beginn an betreibt Bernard eine ironische Relativierung der politischen Positionen, indem er diese als kontingent entlarvt, hinter denen sich letztlich rein egoistische Motive verbergen. Nur weil Béjun eine ehrgeizige Ehefrau, Léontine, hat, die um jeden Preis Bürgermeistersgattin werden will und ihren völlig unambitionierten und darob von ihr verachteten Gatten in einen Wahlkampf 
gegen den adligen Rivalen Baron St-Amour hetzt, wird das Blatt $L a$ Torche überhaupt gegründet. Nur weil der Baron konservativ-reaktionäre Positionen vertritt, muss sich das Blatt somit als links-republikanisches profilieren - die Positionen als solche sind völlig inhaltsleer und beliebig. Dem Streben nach Sozialprestige bei der Gattin korrespondiert die Geldgier beim Gatten. Dieser lässt sich noch im I. Akt seine Zeitung vom Baron für 40000 Fr. abkaufen und verpflichtet sich, als einziger Drucker im Ort, sie zu drucken und zu verteilen - ein finanzielles Geschäft für ihn, das er mit der Dissoziation seiner Person in zwei verschiedene Rollen, die des politischen « adversaire » einerseits und des unpolitischen « imprimeur » und Geschäftsmannes andererseits rechtfertigt. La Torche wechselt damit die politische Farbe und mutiert vom linksradikalen zum rechtsreaktionären Blatt, was die sofortige Gründung einer Gegenzeitung, Le Phare, zur Folge hat. Der Redaktionssekretär Flache, der seinerseits früher bei einem rechten Blatt gearbeitet hat und seit kurzem erst beim linken Blatt arbeitet, geht mit und vollzieht damit in kürzester Zeit ganz unproblematisch seinen zweiten Lagerwechsel. Gegenüber dem erstaunten Baron rechtfertigt er dies als Pflichterfüllung gegenüber seiner Zeitung und als Position der Konstanz, der gegenüber die Differenz der jeweils vertretenen ideologischen Positionen zu einer « question de nuance » wird :

LE BARON - Comment ! Mais, vous consentiriez donc à rester ?

FLACHE - Si je consens ! Mais c'est mon devoir !

LE BARON - Mais la Torche cesse d'être un organe révolutionnaire. Nous allons défendre des idées diamétralement opposées.

FLACHE - Monsieur le baron, me faites-vous l'injure de supposer que, pour une question de nuance, pour une mesquine différence d'opinions... (Se tournant vers Béjun) Je ne suis pas de ceux qui désertent, moi !

BÉJUN - À la bonne heure ! (Au baron) Comme ça, vous pourrez paraître dès demain.

LE BARON - Mais je n'ai pas de texte pour le journal.

FLACHE - Nous avons des articles en retard, tout composés.

BÉJUN - Bien sûr, vos n'aurez qu'à changer les noms, remplacer ceux qu'on engueulait par ceux à qui on passait de la pommade.

LE BARON - En effet, dans ces conditions... (I, 7 ; DC, 9)

Bei Schnitzler ist die Beliebigkeit nicht so extrem, doch auch hier findet sich Entsprechendes. So werden im I. Akt nicht nur die einzelnen Redakteure karikiert, sondern Die Gegenwart erweist sich als ein «Blatt, wo man mit allen Leuten gut ist, die Karriere gemacht haben » (FF, 578), wo die Wahrheit aus Rücksichtnahme auf einzelne Minister oder Staaten schon mal verbogen werden darf, gemäß der von Chefredakteur Leuchter vorgegebenen Devise : «Aber die Wahrheit ist 
etwas sehr Relatives, und jedenfalls läßt sie sich auf verschiedene Arten aussprechen : scharf oder mild » (FF, 575).

Soweit in Kürze die semantisch-ideologische Grundkonstellation, in deren Rahmen in beiden Texten die spezielle Doppelgänger-Problematik des jeweiligen Helden nun entfaltet wird.

\section{Die Gespaltenen : zwischen Psychopathologie und Groteske}

Bei Bernard resultiert die Spaltungs-Konstellation aus einer erotischen Verwicklung. Gélidon verliebt sich in Paris in eine junge Frau, von der er nur den Wohnort, Valmoutiers, nicht aber den Namen weiß. Er reist ihr nach, mietet sich in der Imprimerie von Monsieur Béjun ein und gerät damit in die Intrige. Léontine kommt der Schriftsteller gerade recht. Sie verführt ihn, beginnt ein ehebrecherisches Verhältnis mit ihm und bestimmt ihn, die Position des Chefradakteurs in dem vor kurzem gegründeten Blatt La Torche einzunehmen. Als er bald darauf per Zufall in der Tochter des Barons, Madeleine, die von ihm gesuchte Geliebte wiederfindet, ist dies der Anlass für die Kontaktaufnahme mit dem politischen Gegner, in dessen Blatt er ebenfalls sogleich Chefredakteur wird. Die Folge ist jene « double existence » (II, 5 ; DC, 17), die sein Leben in zwei Hälften teilt : Als Gélidon verbringt er Nacht und Vormittag bei Léontine, als Montillac den Mittag und Nachmittag bei Madeleine und ihrem Vater.

Das Maximum an Relativierung der politischen Überzeugungen wird somit scheinbar beim Protagonisten erreicht, der beide gegensätzlichen Positionen in Personalunion vertritt. Doch der Status dieser Relativierung ist ein fundamental anderer. Im Gegensatz zu den anderen, die aus Kalkül, von Macht- oder Geldinteressen angetrieben, handeln, erscheint der Protagonist als positiviert, denn er als einziger glaubt jeweils an das, was er öffentlich vertritt. Er besitzt eine merkwürdige Eigenart: die erstaunliche Fähigkeit, sich fremden Personen, hier den beiden Partnerinnen, zu assimilieren und seine eigene Position jeweils in maximaler Anverwandlung an deren Positionen zu bestimmen :

MADELEINE - Alors, je compte sur vous. A tout à l'heure. Elle sort.

GÉLIDON, la regardant sortir, extasié - Ah, c'est la vérité ! Voilà la vérité ! Quelle franchise ! Pas de fausse ingénuité ! Elle est exquise. (I, $9.10 ; \mathrm{DC}, 10)$

$[\ldots]$

LÉONTINE - Lucien ! Lucien ! Je t'aime ! [...] (Elle l'embrasse, longuement avec frénésie. Gélidon résiste d'abord, pui s'écrie, exalté)

GÉLIDON - C'est la vérité! Voilà la vérité !

LÉONTINE - Qu'est-ce qui est la vérité? 
GéLIDON - Toi ! Toi ! Rien n'existe au monde que toi... Un homme qui t'a, qui te possède, qui peut te serrer dans ses bras, à toute heure du jour et de la nuit, s'en irait !... Quel fou que cet homme ! quel fou ! (Il l'embrasse avec frénésie) $\mathrm{Ah}$ ! je vais lui servir quelque chose, au baron de Saint-Amour ! (I, 13 ; DC, 13)

Der im Nebentext signalisierte Grad an Emotionalisierung und das artikulierte emphatische Wahrheitspathos bedeuten, dass Gélidon seine öffentliche journalistische Positionierung gleichsam <existentiell auffüllt> und dergestalt Äußeres und Inneres seiner Person restlos zusammenfallen lässt. Diese Eigenart konstituiert aber zugleich auch eine potentiell pathologische Dimension : Indem er zwei Identitäten besitzt, ist er faktisch ich-los. Bernard legt damit ein Problem an, das er aber nicht weiter ausbaut, sondern im harmlosen Rahmen einer erotischen Verstrickung belässt. Schnitzler hingegen entfaltet genau diesen Konflikt nun als systematisches Problem jenseits von erotischen Verwicklungen. Ende 1913 musste ihm die Übereinstimmung zwischen seinem und Bernards Stück umso größer erscheinen, als er selbst die Doppelexistenz seines Helden mit zwei erotischen Verhältnissen verknüpfen wollte. Der gänzliche Wegfall einer erotischen Handlung, der erst relativ spät erfolgte ${ }^{11}$ und somit kaum als Reaktion auf Bernard zu deuten ist, ergibt einen der auffälligsten Unterschiede. Das Fehlen einer erotischen Dimension ist freilich konstitutiv und wird im Text selbst thematisiert (s.u.).

Auch hier ist das zentrale Merkmal des Helden seine Fähigkeit zur maximalen Anverwandlung fremder Positionen. Bereits seit einem Jahr ist Fliederbusch Parlamentsberichterstatter bei der Gegenwart und schreibt langweilige Berichte von den politischen Debatten, von den Kollegen getadelt als « stenographische Protokoll[e] » ohne «persönliche Note » und «Stellungnahme» (FF, 565). Seine plötzliche Wandlung wird ausgelöst durch den Redaktionskollegen Füllmann, der ihm die von Fink im gegnerischen Blatt geschriebene Berichterstattung über dieselbe Parlamentsdebatte vorliest - eine Lektüre, die als Katalysator für eine - scheinbare - journalistische Selbstfindung fungiert. Erst durch die emotional aufgeladene Schilderung derjenigen Parlamentssitzung, der er selbst beigewohnt hat, und nur über den Umweg über die fremde Entrüstung kann die eigene ausgelöst werden, die dann in den Schreibakt mündet. Analog zu Bernard notiert Schnitzler im Nebentext : « immer stärker interessiert », "wachsende Erregung in seinen Zügen » (FF, 572f.). Die Situation im I. Akt in der Redaktion der Gegenwart wiederholt sich im II.Akt in der Redaktion der Eleganten Welt, wo Fliederbusch

11. - Siehe Cambridger Nachlass, Mappe A 113,1-4, datiert vom Dez. 1912 bis Dez. 1913. 
zunächst «leuchtend » den politischen Stellungnahmen des Grafen folgt, um sich sodann, allein, immer mehr in die Gegnerschaft gegen seinen eigenen für die Gegenwart geschriebenen Text, den er sich laut vorliest, hineinzusteigern (FF, 597, 599f.). Gegenüber Bernard ist das Problem radikalisiert, insofern der Held von Anfang an und ohne jegliche Motivation über Frauengeschichten als gespalten erscheint. Die entworfene Konstellation ist paradox : In dem Maße, wie der Held - als Fliederbusch $-\mathrm{zu}$ sich findet, in einer dezidierten politischen Position, entfernt er sich zugleich von sich selbst - als Fink - und vice versa, bis zu dem Grad, dass er seine eigenen Produkte nicht mehr kennt. Selbstfindung und Selbstentfremdung bzw. -verlust bedingen einander. Die Problematik des scheinbaren Opportunisten wird hier als gleichsam existentielle gestaltet und erweist sich damit als grundlegend transformiert, weshalb Fliederbusch denn auch als zweiter « Schmock » nicht adäquat zu fassen ist.

Seit ihrer Erfindung um 1800 steht die literarische Spaltungs- bzw. Doppelgängerproblematik in einer charakteristischen - historisch je spezifischen - Spannung zwischen Okkultismus bzw. Fantastik und Psychopathologie ${ }^{12}$. Je realistisch-mimetischer die literarische Darstellung operiert, desto mehr nähert sie sich logischerweise der reinen psychopathologischen Studie an, wie etwa Paul Lindaus <naturalistischer> Einakter Der Andere (1893) demonstriert. Bernard spielt mit dieser Dimension, wenn er seinen Helden gegenüber beiden Partnerinnen, die Aufklärung wegen seiner Absenzen begehren, anführen lässt, er leide unter Neurasthenie. Hinter der vordergründigen Ausrede (vgl. die Begründung gegenüber dem Freund: «Cette maladie-là n'a rien de dégoûtant et les signes extérieurs en sont vagues » II, 5 ; DC, 17) verbirgt sich freilich ein - von Bernard im Gegensatz zu Schnitzler nicht weiter problematisierter - Konflikt. Schnitzler wählt keine dieser beiden Optionen, sondern stattdessen einen dritten Weg, nämlich die Variante der humoristischen Groteske, die qua Gattungskonvention eine Lizenz für gewisse Unwahrscheinlichkeiten erteilt. Schnitzler, so meine Hypothese, geht es weniger um ein primär psychopathologisches Problem (allenfalls in einem abstrakt-zeichenhaften Sinn), auch nur vordergründig um so etwas wie Pressekritik ${ }^{13}$, vielmehr um ein quasi (sozial) philosophisches Problem von Identität und Authentizität im Kontext der Weltanschauungsproblematik.

12. - Siehe die Studie von Andrew J. Webber: The Doppelgänger. Double Visions in German Literature. New York, 1996; vgl. auch Sabler, a.a.O., S. 324-327.

13. - Hierzu auch Ernst Offermanns : «Héro du jour - et déchu : quelques aspects du personnage de Mimosas dans la comédie Les Journalistes d'Arthur Schnitzler », in : Le Rider/Wentzig, a.a.O., S. 72-84, hier 74-77. 
Signifikant erscheint in diesem Zusammenhang eine wiederholt thematisierte und, soweit ich sehe, bislang kaum wahrgenommene, spezifische Art des 〈Realitätsbezugs〉 des Helden. Die unmittelbare empirische Realität als solche vermag nämlich offenbar keinerlei Selbstpositionierung und emotionale Wirkung bei Fliederbusch hervorzurufen, wie exemplarisch bei der Parlamentsrede des Grafen der Fall :

FLIEDERBUSCH - Entschuldigen Sie, Herr Füllmann, ich habe der Sitzung beigewohnt, - ich erinnere mich nicht, daß der Graf Niederhof diese Worte -

FÜLLMANN - Der Sinn seiner Worte war es, junger Mann ! [...]

$[\ldots]$

FLIEDERBUSCH anfangs zögernd, dann immer bestimmter - Vor einer Weile hat Herr Füllmann einiges aus dem Artikel der »Eleganten Welt«, auf den ich mich beziehe, hier vorgelesen. Ich hatte ihn zwar schon gekannt [...]. Aber ich muß gestehen, beim flüchtigen Lesen war er ziemlich spurlos an mir vorübergegangen. Erst als Herr Füllmann die markantesten Stellen vortrug, im Ton edelster und gerechtfertigter Entrüstung [...] Da offenbarte sich mir allmählich von Wort zu Wort die ganze Zweideutigkeit, die ganze Niederträchtigkeit der Gesinnung, die darin zum Ausdruck kommen. - Die Parlamentssitzung, in der Graf Niederhof das Wort ergriff, tauchte plötzlich wieder vor mir auf. Ich sah den Grafen vor mir stehen in seiner ganzen feudalen Überhebung - Sie hätten ihn sehen müssen, meine Herren ! - Meine Empörung war grenzenlos. Ich glaube, ich wäre daran erstickt, wenn ich mich nicht sofort hingesetzt und eine Art Entgegnung verfaßt hätte. (FF, 570, 580f., Hervorh. WL)

Wie diese Rede erst in der verbalen Vermittlung durch Fremde wirkt, so schreibt Fliederbusch nach seiner Wandlung zum profilierten Journalisten in auffälliger Weise über Objekte und Themen, die er selbst gar nicht aus eigener Anschauung kennt : so exemplarisch sein großer Artikel über Park und Bildergalerie der Fürstin, Cousine und Ex-Geliebte des Grafen. Wie wiederum mehrfach betont wird, kennt er die Gegenstände seines Artikels ausschließlich als « Reproduktion » und aus Photographien (FF, 606f., 610, 625). Die semiotische - sei es verbale, sei es ikonische - Repräsentation von Wirklichkeit genügt bereits, um seine Produktion anzuregen, ja sie substituiert ihm nicht nur die Realität, sondern ist gewissermaßen mehr als diese selbst.

Schnitzler entwirft mit dem Redaktionskollegen Styx eine zu Fliederbusch komplementäre Figur. Dieser, der die Doppelexistenz des Helden ansatzweise durchschaut, lebt selbst eine solche, allerdings sukzessiv, nicht simultan. Einstmals adliger Offizier, aufgrund Spielschulden unehrenhaft entlassen, führt er jetzt eine zivile Existenz und praktiziert als Journalist das Gegenmodell zum Helden: Er stellt nämlich, wie er mehrfach hervorhebt, empirische «Recherchen » an, so dass seine Schränke vor « Material » «bersten » (FF, 588, 600, 615). 
Während Fliederbusch mit semiotischen Substituten arbeitet und «aus der Phantasie » (FF, 610) schöpfen muss, kennt Styx seine Gegenstände, Personen und Orte, aus eigener unmittelbarer Anschauung.

Fliederbusch repräsentiert also keineswegs den Gipfel einer allgemein herrschenden Überzeugungslosigkeit als Ausdruck einer epochalen Wertekrise, wie teilweise behauptet wird ${ }^{14}$; im Gegenteil, er, der nicht zu wenig, sondern 〈zu viel〉 Überzeugung besitzt, scheint in ironischgrotesk übersteigerter Form die Gefahr einer «falschen Antwort $>$ auf diese Situation zu demonstrieren. Greifbar wird dies bei der Konfrontation mit seinem Hauptgegenspieler, dem Grafen Niederhof, die im III. Akt, kurz vor dem anberaumten Duell, in der zentralen Dialogszene des Dramas entfaltet wird. Die Kontroverse über Sinn und Notwendigkeit dieses Duells mündet schnell in die prinzipielle Frage, wie sich sozial wahrnehmbare Handlungen (auch Sprachhandlungen) einerseits und das unsichtbare Innere der Person, ihre Gefühle, Einstellungen, Überzeugungen andererseits, kurzum, wie sich Worte und Wesen eines Individuums zueinander verhalten. Wie Fliederbusch nicht zwischen semiotisch repräsentierter und faktischer Realität unterscheidet, so identifiziert er auch die Rolle, die eine Person in der Öffentlichkeit spielt, ihre Worte und « symbolische Bedeutung » « restlos » mit ihrem innersten «Wesen » (FF, 628, 630). Aus dieser Ontologisierung folgt logisch konsequent der Zwang, für seine ideologische Überzeugung mit dem eigenen Leben einzustehen und «Blutzeugenschaft » abzulegen (FF, 628). Demgegenüber besteht der Graf auf einer grundsätzlichen Unterscheidung und Dissoziation beider Ebenen. Er formuliert eine skeptische Position, die nicht nur zu relativieren vermag, sondern so weit geht, die Existenz von « Überzeugungen » überhaupt, als kontingente Produkte und als Selbsttäuschung, in Frage zu stellen. So sehr er sonst selbst Objekt der satirischen Verspottung in diesem Stück ist - diesbezüglich formuliert er zweifellos ranghöchste autorkonsensuelle (und «moderne ! ${ }^{15}$ ) Positionen :

GRAF kopfschüttelnd - Zu einem solchen Grad von - Erbitterung seh ich doch eigentlich keinen Anlaß. Herr Fliederbusch vertritt am Ende doch nur se ine n Standpunkt, wie Sie den Ihrigen, Herr Fink. Im Ausdruck mag er ja stellenweise etwas zu weit gegangen sein; aber sein S tand punkt hat schließlich geradeso seine Berechtigung - wie der Ihrige. [...] Für Sie, Herr Fink, kommt selbstverständlich nur die politische Idee in Betracht, als deren Repräsentanten Sie am Ende zwei Dutzend andre meiner Parteigenossen geradeso betrachten können wie mich. Aber wer sagt Ihnen überhaupt, daß die Person des Grafen

14. - So Weinberger a.a.O., S. 57. Vgl. hierzu auch, differenzierter, Offermanns, «Héros du jour - et déchu », a.a.O., S. 73f.

15. - Vgl. Zieger, a.a.O., S. 304. 
Niederhof mit dem Symbol etwas zu tun hat, das er zufällig für Sie bedeutet $-[\ldots]$ ? (FF, 628; Hervorh. i. O.)

Das Phänomen der 〈Relativierung〉 wird nicht nur in Bezug auf politische Positionen relevant, sondern auch hinsichtlich des gelebten Lebensentwurfs. Protagonist und Graf repräsentieren nämlich auch zwei oppositionelle Personkonzepte. Die moderne zeitgenössische Personbzw. Subjektkonzeption postuliert, dass jedes Subjekt ein Potential an noch nicht Gelebtem in sich birgt, gleichsam eine Pluralität möglicher Subjektentwürfe, deren Bewusstwerdung und zumindest partielle Realisierung dem Subjekt aufgetragen ist ${ }^{16}$. Der Graf umfasst nun tatsächlich eine ganze Serie von gleichsam verschiedenen 〈Personen〉, d.h. Existenzen, Rollen, Berufen etc., die er in seinem Leben absolviert hat. Angespielt wird u.a. auf eine frühere Militärlaufbahn, mit Liebesaffären und Duellen. Politik und Journalismus, so heißt es zwar nicht ohne Ironie, doch ohne einen Hauch von moralischer Verurteilung, sei « das einzige, was er noch nicht probiert [habe] ( FF, 571, 605, 632). Fliederbusch/Fink hingegen kennt nur seinen aktuellen Beruf, sonst nichts - explizit auch kein erotisches Privatleben, wie er der Fürstin, die annimmt, er müsse doch vor seinem Duell noch Wichtigeres zu erledigen haben, als mit ihr über ihre Ahnengalerie zu plaudern, etwa den Abschied von der Geliebten oder von der Familie, gesteht : « Ich habe nichts als meinen Beruf, Durchlaucht, [...]. » (FF, 609) Während der Graf also verschiedene Identitäten sukzessive in sein Ich integriert und eine differenzierte, reiche Persönlichkeit darstellt, ist der Titelheld desintegriert und zerfällt in zwei antagonistische simultane Identitäten.

Schnitzler entwirft hinsichtlich des Problems von 〈Authentizität> und 〈Wahrheit〉 also eine Skala mit zwei Extrempositionen : am einen Pol die maximale Inkongruenz zwischen 〈Innen〉 und 〈Außen〉 bei den Heuchlern, den Feigen und Verlogenen, wie sie in beiden Zeitungen und beiden politischen Lagern auftreten - am anderen Pol die maximale Kongruenz beider Dimensionen in der grotesken Zuspitzung des naiven Protagonisten. Was scheinbar zu Selbstfindung und 〈Wahrheit> führt, ist faktisch einem Realitäts- sowie psychischen Selbstverlust äquivalent. Beide Positionen sind gleichermaßen negativ und werden dem Spott preisgegeben. Als die optimale Position resultiert eine der Mitte : die des Grafen, der um die Unmöglichkeit einer Kongruenz weiß, mit dieser Desillusionierung als « Sportsman » (FF, 632f.) umgehen kann,

16. - Siehe Michael Titzmann : « Das Konzept der 〈Person〉 und ihrer 〈Identität〉 in der deutschen Literatur des frühen 20. Jahrhunderts », in: Manfred Pfister (Hg.) : Krise und Modernisierung des Ich um 1900, Passau, Rothe, 1989, S. 36-52 ; W. Lukas : Das Selbst und das Fremde. Epochale Lebenskrisen und ihre Lösung im Werk Arthur Schnitzlers, München, Fink, 1996, Kap. 1.122 «Zur neuen Psychologie und Personkonzeption». 
indem er sie reflektiert und genau dadurch eine Art 〈höherer Moral〉 gewinnt. Die Konfrontation mit dem Grafen erhält somit für den Helden auch eine quasi-therapeutische Funktion, weshalb das Ende dieses Stücks keineswegs im düsteren (Kultur) Pessimismus verharrt ${ }^{17}$. Der Ausgleich der Gegensätze, der am Ende symbolisch inszeniert wird und mit dem die Komödie ihren narrativen Zielpunkt erreicht, hat denn auch nichts mehr mit jenem feigen Ausgleich zu tun, den der Chefredakteur der Gegenwart zu Textbeginn " als die vornehmste Aufgabe eines wahrhaft demokratischen Organs » ausgibt (F, 575). Vielmehr werden jetzt erst, auf der Basis eines skeptischen Humanismus und auf einer Metaebene, die weltanschaulichen Gegensätze völlig neutralisiert und überwunden ${ }^{18}$.

Im Unterschied zu Bernard, der im III. Akt eine farcenhafte Auflösung inszeniert, die, ganz gattungskonform, in die Heirat des jungen Liebespaares mündet, verfolgt Schnitzler ein ganz anderes Ziel. Er entwickelt aus der identischen Ausgangssituation, die sich, wie gezeigt, keineswegs nur auf Pressesatire beschränkt, eine geradezu hochphilosophische und artistisch ausgefeilte Komödie. Sie versucht, differenzierte und ernsthafte Antworten auf das zeitgenössisch virulente Problem ideologischer Radikalisierung zu geben und zählt gleichwohl, oder gerade deswegen, zu seinen besten komischen Stücken.

17. - So Weinberger, a.a.O. und Le Rider, a.a.O.

18. - $\mathrm{Zu}$ diesem von Schnitzler in zahlreichen erzählenden und dramatischen Werken narrativ entfalteten Denkmodell s. W. Lukas, a.a.O. 
